\title{
DETERMINAÇÃO DA PRODUTIVIDADE DA ÁGUA COM IMAGENS LANDSAT 8 NA REGIÃO SEMIÁRIDA DO BRASIL
}

\author{
Antônio Heriberto de Castro TeiXeira, Janice Freitas Leiva \\ Embrapa Monitoramento por Satélite
}

<heriberto.teixeira@embrapa.br>, <janice.leivas@embrapa.br>

DOI: $10.21439 /$ conexoes.v11i1.1064

\begin{abstract}
Resumo. Considerando o cenário de mudança de uso da terra aliada às alterações climáticas no semiárido brasileiro, a evapotranspiração atual (ET), a produção de biomassa (BIO) e a produtividade da água (PA) baseada na ET, foram quantificadas sob diferentes condições termo hídricas com três imagens Landsat 8 (L8) de 2014 no município de Juazeiro, Estado da Bahia (BA), Nordeste do Brasil. Os valores médios dos pixels para ET, BIO e PA, nas culturas irrigadas, variaram respectivamente de $2.9 \pm 1.2$ a $3.8 \pm 1.6 \mathrm{~mm}$ $\mathrm{d}^{-1} ; 107 \pm 64$ a $124 \pm 75 \mathrm{~kg} \mathrm{ha}^{-1} \mathrm{~d}^{-1} ;$ e de $3.0 \pm 0.7$ a $3.4 \pm 0.8 \mathrm{~kg} \mathrm{~m}^{-3}$. As faixas correspondentes para a Caatinga foram de $0.1 \pm 0.1$ a $0.9 \pm 0.6 \mathrm{~mm} \mathrm{~d}^{-1} ; 1 \pm 2$ a $13 \pm 11 \mathrm{~kg} \mathrm{ha}^{-1} \mathrm{~d}^{-1}$, e $0.5 \pm 0.6$ a $1.4 \pm 0.5 \mathrm{~kg} \mathrm{~m}^{-3}$. Os valores incrementais da PA, representativos da substituição da vegetação natural por culturas irrigadas foram de $300 \%$, para o ano estudado. Maior uniformidade nos parâmetros hídricos e de vegetação ocorreu nas espécies da Caatinga, evidenciada pelos menores valores de desvio padrão quando comparados com aqueles para as culturas agrícolas. Os modelos testados no atual estudo podem subsidiar o monitoramento dos agros-ecossistemas, sendo valiosas ferramentas para a sustentabilidade dos recursos hídricos na região semiárida do Brasil.
\end{abstract}

Palavras-chaves: Evapotranspiração. Produção de biomassa. Mudança de uso da terra.

\begin{abstract}
Considering the climate and land-use change scenario in the Brazilian semi-arid region, actual evapotranspiration (ET), biomass production (BIO), and water productivity (WP) based on ET, were quantified with Landsat 8 images under different thermo-hydrological conditions of 2014, in the Juazeiro municipality, Bahia (BA) state, Northeast Brazil. The mean pixel values for ET, BIO, and PA, for irrigated crops, ranged respectively from $2.9 \pm 1.2$ to $3.8 \pm 1.6 \mathrm{~mm} \mathrm{~d}^{-1} ; 107 \pm 64$ to $124 \pm 75 \mathrm{~kg} \mathrm{ha}^{-1} \mathrm{~d}^{-1}$; and from $3.0 \pm 0.7$ to $3.4 \pm 0.8 \mathrm{~kg} \mathrm{~m}^{-1}$. The corresponding ranges for "Caatinga" were $0.1 \pm 0.1$ to 0.9 $\pm 0.6 \mathrm{~mm} \mathrm{~d}^{-1} ; 1 \pm 2$ to $13 \pm 11 \mathrm{~kg} \mathrm{ha}^{-1} \mathrm{~d}^{-1}$; and $0.5 \pm 0.6$ to $1.4 \pm 0.5 \mathrm{~kg} \mathrm{~m}^{-3}$. The incremental PA values, representing the consequence of replacement of natural vegetation by irrigated crops were $300 \%$ for the studied year. Higher uniformity for the water and vegetation parameters happened in "Caatinga" species, evidenced by the lower values of standard deviation, when compared with those for agricultural crops. The models tested in the current study can subsidize the monitoring of the agro-ecosystems, being powerful tools for the sustainability of the water resources in the Brazilian semi-arid region.
\end{abstract}

Keywords: Evapotranspiration. Biomass production. Land-use changes.

\section{INTRODUÇÃO}

No semiárido brasileiro, a disponibilidade dos recursos hídricos para irrigação é responsável pelo crescimento da economia rural; entretanto, uma das grandes consequências deste desenvolvimento é que outros usuários da água estarão competindo com o setor agrícola (TEIXEIRA, 2009). Nestas condições, a fotossíntese e a produção de biomassa (BIO) podem agir como consu- midores de carbono, afetando as trocas energéticas entre as superfícies vegetadas e a baixa atmosfera (CESCHIA et al., 2010).

O município de Juazeiro, na região semiárida do Estado da Bahia, Nordeste do Brasil, é atualmente um importante polo agrícola, como consequência do desenvolvimento de tecnologias de irrigação, principalmente em fruteiras comerciais, nas vizinhanças do Rio 
DETERMINAÇÃO DA PRODUTIVIDADE DA ÁGUA COM IMAGENS LANDSAT 8 NA REGIÃO SEMIÁRIDA DO BRASIL

São Francisco. Nos agros-ecossistemas mistos, o uso do sensoriamento remoto por imagens de satélites, para a quantificação dos componentes da produtividade da água (PA) em larga escala se torna muito relevante. Um melhor conhecimento da PA dos diferentes tipos de vegetação prover informações valiosas para o atingimento das práticas de conservação dos recursos hídricos sem a perda da produção agrícola. Para atingir este objetivo, deve-se fazer a quantificação da evapotranspiração atual (ET) e da produção atual $\left(Y_{a}\right)$ em larga escala.

A ET é criticamente importante por ser essencial para a produção vegetal e o seu aumento implica em menos água disponível para os usos ecológicos e humanos nas bacias hidrográficas. As dificuldades da sua obtenção em larga escala através de medições de campo estimularam o uso do sensoriamento remoto por imagens de satélites (TANG; ROSENBERG; LETTENMAIER, 2009; TEIXEIRA, 2010; MIRALLES et al. 2011; PÔÇAS et al. 2013; TEIXEIRA et al., 2014 LEIVAS et al., 2016).

Objetivando a extrapolação de medições pontuais na região semiárida brasileira, a ET tem sido estimada por meio dos satélites Landsat 5 and 7 com aplicação do algoritmo SEBAL (Surface Energy Balance Algorithm for Land) desenvolvido por Bastiaanssen et al. (1998) através de processos de calibrações e validações (TEIXEIRA et al. 2009a, TEIXEIRA et al., 2009b).

Embora em geral o SEBAL tenha apresentado bons resultados no semiárido brasileiro, o problema reside na necessidade de identificação de condições hidrológicas extremas, não sendo realístico desprezar a ET na estação chuvosa, quando as condições de umidade do solo nos diferentes agros-ecossistemas, constituídos de vegetação natural e culturas irrigadas, são homogêneas. Outro problema com relação a alguns modelos para estimativa da ET com imagens de satélites, quando se almejam os usuários finais, é a necessidade de conhecimentos específicos da física da radiação. Como alternativas mais simples, a viabilidade de aplicação da equação de Penman-Monteith (PM) tem sido demonstrada com o uso de índices de vegetação obtidos por sensoriamento remoto em conjunto com dados climáticos (CLEUGH et al., 2007; NAGLER et al., 2013, KAMBLE; KILIC; HUBBARD, 2013).

Considerando a simplicidade de aplicação, e a não necessidade de condições hidrológicas extremas, não sendo necessária a determinação de pixels frios e quentes na imagem, como no caso do SEBAL, o algoritmo SAFER (Simple Algorithm For Evapotranspiration Retrieving) foi desenvolvido e validado na região semiárida do Brasil através de medições simultâneas em campo com quatro torres de balanço de energia e imagens Landsat, envolvendo fortes contrastes de condições termo hídricas ao longo de vários anos (TEIXEIRA et al., 2008; TEIXEIRA, 2010; TEIXEIRA et al., 2013; TEIXEIRA et al., 2014).

O sensoriamento remoto através de imagens de satélites é também uma ferramenta efetiva para a estimativa da $\mathrm{BIO}$ em larga escala. O modelo da radiação proposto por Monteith (1972) tem precisão aceitável para este fim, provendo informações espaciais e temporais da localização e do estado da vegetação. Com a determinação da ET através do SAFER e estimativas da energia disponível, introduz-se o efeito das condições de umidade nas variações da BIO das diferentes estações do ano (TEIXEIRA et al., 2013).

Um terceiro modelo para calcular a resistência da superfície aos fluxos hídricos $\left(r_{s}\right)$, o SUREAL (Surface Resistance Algorithm) é usado (TEIXEIRA et al. 2014). Este modelo, no atual trabalho foi aplicado para a separação das variáveis hídricas e de vegetação das culturas irrigadas e Caatinga nas condições semiáridas do município de Juazeiro. O SUREAL também foi elaborado e validado com as mesmas medições simultâneas de campo e por satélites usadas no caso do SAFER.

A PA pode ser definida como a razão dos benefícios líquidos de uma cultura, floresta, pesca, pecuária, e culturas consorciadas pela quantidade de água para produzir aqueles benefícios. Considerando a vegetação, esta pode ser a BIO pela água consumida, incluindo aquela originada das chuvas, irrigação, infiltração e mudanças no armazenamento do solo (MOLDEN et al., 2007). Considera-se a produtividade da água de culturas (PAC) como a razão da $Y_{a}$ para a quantidade de água consumida ou aplicada. Muitos caminhos existem para melhorias da PAC na agricultura, tanto na dependência de chuvas, como irrigada (TEIXEIRA, 2009).

Embora os modelos SAFER, da radiação de Monteith e o SUREAL tenham sido usados com sucesso em imagens Landsat 5/7 e MODIS na região semiárida do Brasil (TEIXEIRA et al. 2014), estes ainda não foram testados com imagens Landsat 8 (L8). O satélite L8 foi lançado em 11 de fevereiro de 2013, com as operações normais se iniciando em 30 de maio de 2013. Com um tempo de revisita de 16 dias, cruzando o equador às 10:00 hr, o sensor L8 tem 9 bandas com uma varredura de $185 \mathrm{~km}$ (DIJK; BRUIJNZEEL; SCHELLEKENS, 2004). Portanto, novas pesquisas são necessárias com o uso do L8 para avaliação da combinação dos modelos da ET e da BIO, especialmente para aplicações em agros-ecossistemas mistos, com elevada heterogeneidade termo hídrica, espacial e temporal.

Este trabalho destaca a combinação dos modelos 
DETERMINAÇÃO DA PRODUTIVIDADE DA ÁGUA COM IMAGENS LANDSAT 8 NA REGIÃO SEMIÁRIDA DO BRASIL

SAFER, Monteith e SUREAL (TEIXEIRA et al. 2013. TEIXEIRA et al., 2014) para demonstrar que as medições das bandas do visível, infravermelho próximo e termais do satélite L8, em conjunto com dados agrometeorológicos, podem ser usadas para análises da PA em larga escala nas culturas irrigadas e na Caatinga nas condições semiáridas do município de Juazeiro, Nordeste do Brasil. O sucesso da modelagem realizada no atual estudo com o novo satélite fornece mais confiança para os testes e validações em outras condições semiáridas no mundo, onde provavelmente necessitarão apenas de ajustes nas equações originais.

\section{MATERIAL E MÉTODOS}

\section{1 Área de estudo e dados utilizados}

A Figura 1 apresenta as localizações do município de Juazeiro, Estado da Bahia (BA), Nordeste do Brasil, e das sete estações agrometeorológicas utilizadas para a interpolação dos parâmetros climáticos através de um Sistema de Informações Geográficas (SIG).

Os dados climáticos de entrada (setas pretas na Figura 1] foram da rede de estações da Empresa Brasileira de Pesquisa Agropecuária (Embrapa). Dados da radiação solar global incidente $\left(R_{G}\right)$, temperatura do ar $\left(T_{a}\right)$, umidade relativa (UR) e velocidade do vento $(\mathrm{u})$, foram usados para o cálculo da evapotranspiração de referência $\left(E T_{0}\right)$ pelo método de Penman-Monteith (ALLEN et al., 1998). Os dados diários de $R_{G}, T_{a}$ e $E T_{0}$ foram interpolados pelo método do movimento da média, resultando em grades com pixels de $30 \mathrm{~m}$, compatíveis com os parâmetros obtidos por sensoriamento remoto com as imagens Landsat 8.

O município de Juazeiro (BA) é localizado na região semiárida do Nordeste do Brasil, no Submédio São Francisco. Nesta sub-bacia, a vegetação natural, Caatinga é o tipo de vegetação predominante, a qual tem sido substituída rapidamente por fruteiras irrigadas nas margens do Rio São Francisco. Correntes do Sul, Norte, Leste e Oeste influenciam a climatologia da região. Com exceção das áreas de altitudes elevadas, em geral, a região apresenta normais anuais de temperatura do ar $\left(T_{a}\right)$ acima de $24{ }^{\circ} \mathrm{C}$. A média dos valores máximos é $33{ }^{\circ} \mathrm{C}$ e dos mínimos é $19{ }^{\circ} \mathrm{C}$. Os meses mais quentes são outubro e novembro, quando o Sol está na posição zenital, enquanto os mais frios são junho e julho no solstício de inverno do hemisfério Sul. A homogeneidade térmica contrasta fortemente com a heterogeneidade espacial e temporal do regime pluviométrico. A maioria das chuvas cai durante o primeiro quadrimestre do ano, representando $68 \%$ da quantidade anual, cujo total médio histórico é de $550 \mathrm{~mm}^{2} \mathrm{ano}^{-1}$

\section{(TEIXEIRA, 2009).}

Principalmente fruteiras compõem os cultivos agrícolas irrigados, na maioria videiras, mangueiras, goiabeiras e bananeiras, circundadas pela Caatinga. As espécies deste último ecossistema são caracterizadas por vegetação escassa e senescente no período mais seco do ano, entretanto, tão logo a estação chuvosa se inicia, as plantas se tornam rapidamente verdes. Algumas delas têm a habilidade de armazenamento de água no período mais seco, com graus variáveis de tolerância ao estresse hídrico (TEIXEIRA, 2009).

Dados climáticos foram usadas em conjunto com três imagens do satélite Landsat 8 envolvendo diferentes condições termo hídricas ao longo do ano de 2014 para os dias julianos - DJ 025 (25 de janeiro), 153 (02 de junho) e 265 ( 22 de setembro). Os dias analisados representam, respectivamente, condições dentro do período chuvoso, logo após este período e nas condições naturalmente mais secas do semiárido brasileiro.

\subsection{Modelagem da produtividade da água}

A Figura 2 apresenta o fluxograma para a obtenção da PA em larga escala, com o uso das imagens L8 e dados agrometeorológicos através da combinação do algoritmo SAFER e do modelo da radiação de Monteith.

As parametrizações envolvidas na Figura 2 foram realizadas no semiárido do Brasil através de medições simultâneas em campo e por satélites, envolvendo fortes contrastes agros-ecossistemas e condições termo hídricas contrastantes através de diferentes anos (TEIXEIRA et al., 2008; TEIXEIRA et al., 2014).

Para o cálculo do albedo da superfície $(\alpha 0)$ através do satélite L8, foram usadas as bandas de 1 a 7 , com resolução espacial de $30 \mathrm{~m}$, enquanto que para a temperatura da superfície $\left(T_{0}\right)$ utilizaram-se as bandas termais 10 e 11, com resolução espacial de 100 m.

Seguindo a Figura 2 dos números digitais (ND):

$$
R_{e s p \_b}=a N D+b
$$

onde a e b são os coeficientes de conversão fornecidos no arquivo dos meta dados (VANHELLEMONT; RUDDICK, 2014). O albedo planetário para cada banda do satélite L8 $\left(\alpha p_{b}\right)$ foi calculado como:

$$
\alpha p_{b}=\frac{R_{e s p \_b} \pi d^{2}}{R a_{b} \cos \phi}
$$

onde $R_{e s p \_b}$ é a radiação espectral para os comprimentos de onda da banda $b\left(W m^{-2} s r^{-1} \mu m^{-1}\right)$, d é a distância relativa Terra-Sol; $R a_{b}$ é a radiação atmosférica média para cada banda $\left(W m^{-2} \mu m^{-1}\right)$ e $\phi$ é o ângulo zenital (TEIXEIRA et al., 2014). 


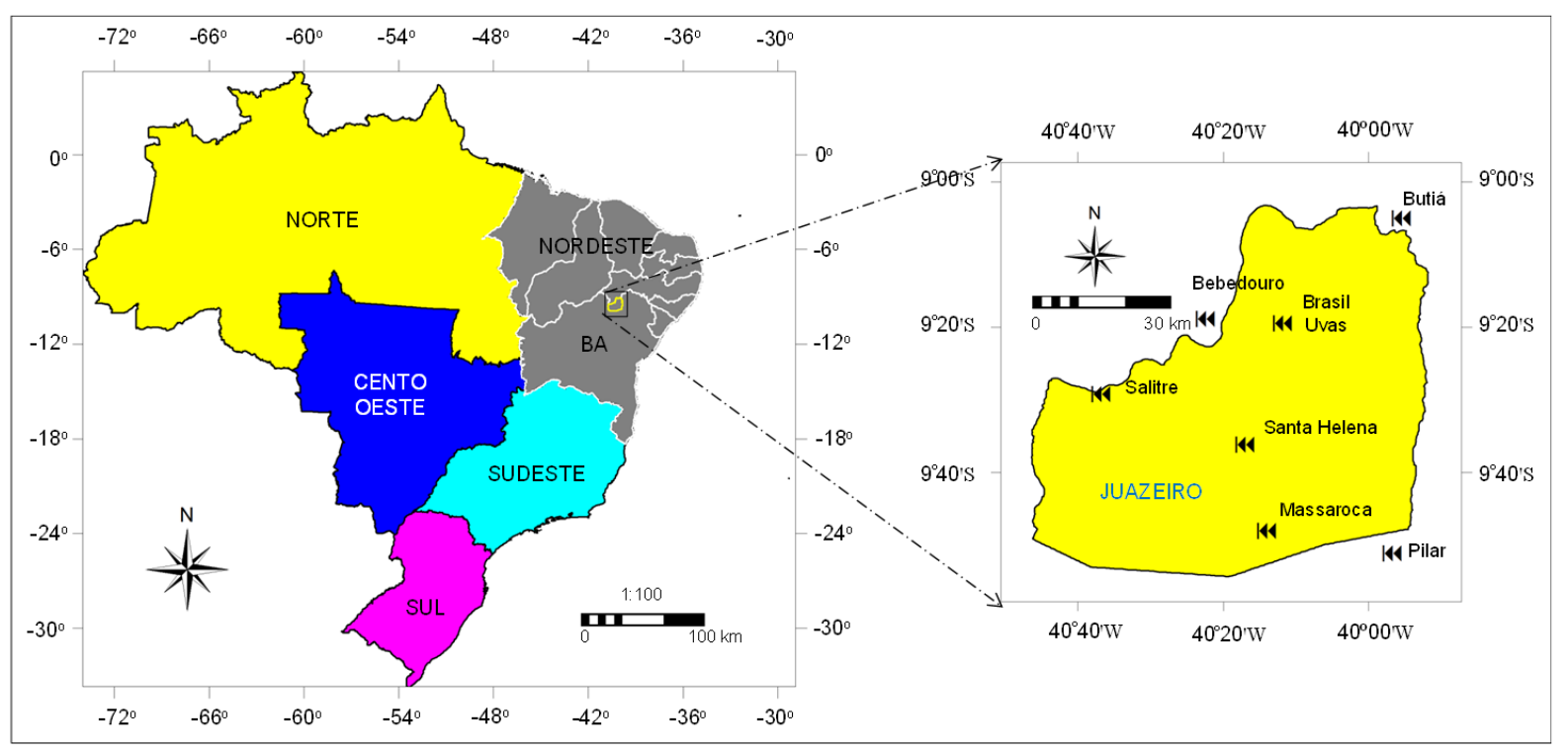

Figura 1: Localização do município de Juazeiro, Estado da Bahia (BA), Nordeste do Brasil, e estações agrometeorológicas (setas pretas) utilizadas para o processo de interpolação dos parâmetros climáticos.

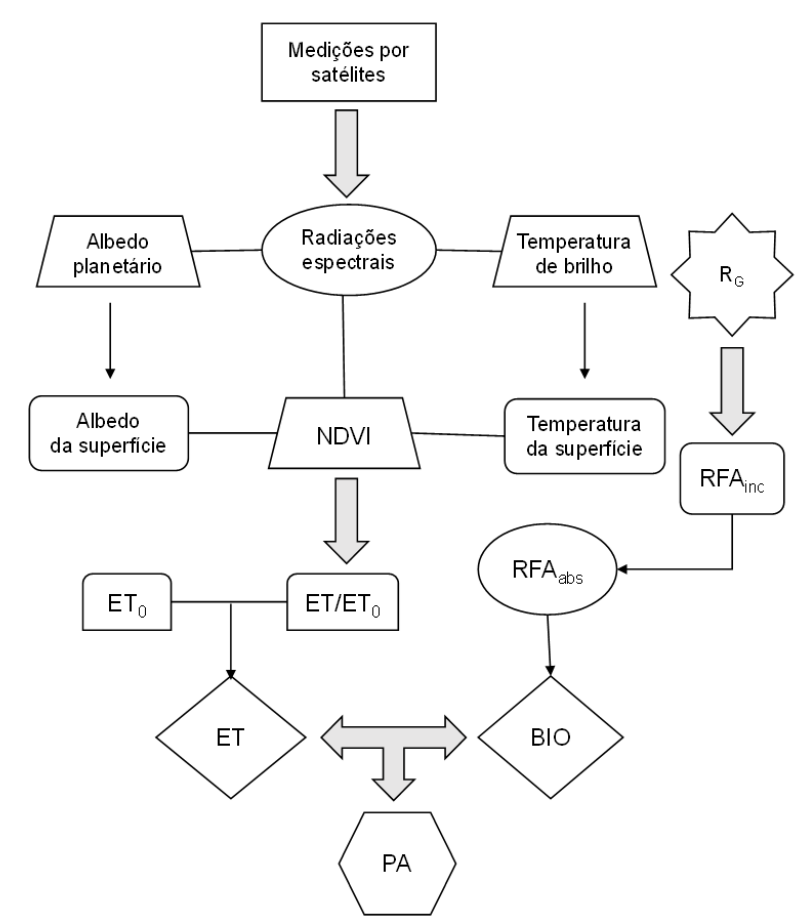

Figura 2: Fluxograma para a obtenção da produtividade da água (PA) em larga escala, através da da combinação do algoritmo SAFER (Simple Algorithm for Evapotranspiratio Retrieving) com o modelo da radiação de Monteith. 
DETERMINAÇÃO DA PRODUTIVIDADE DA ÁGUA COM IMAGENS LANDSAT 8 NA REGIÃO SEMIÁRIDA DO BRASIL

$R a_{b}$ para cada uma das bandas de 1 a 7 do L8 foram calculadas de acordo com a lei de Planck, integrando a radiação nos comprimentos de onda para cada intervalo e considerando a fração sobre o espectro solar, assumindo-se o Sol como um corpo negro. Então o albedo planetário para todo o espectro solar $(\alpha p)$ foi considerado a soma total dos diferentes valores de $\alpha p_{b}$ de acordo com o peso para cada banda:

$$
\alpha p=\sum w_{b} \alpha p_{b}
$$

onde $w_{b}$ é a razão da radiação solar incidente no topo da atmosfera em uma banda particular, para a soma da radiação de todas as bandas.

A Tabela 1 apresenta os comprimentos de onda, $R a_{b}$ e $w_{b}$ para cada uma das bandas utilizadas (B1-B7) no cálculo de $\alpha_{p}$ com as medições do L8.

As radiações espectrais das bandas $10\left(R_{e s p_{1} 0}\right)$ e 11 $\left(R_{e s p_{1} 1}\right)$ das regiões termais do L8 foram convertidas em temperaturas aplicáveis no topo da atmosfera $\left(T_{b}\right)$ pela inversão da lei de Planck no intervalo de comprimento de onda de 10,6-11,19 $\mu \mathrm{m}$ (b10) e 11,5-12,51 $\mu m$ (b10):

$$
T_{b}=\frac{K_{2}}{\ln \left(\frac{K_{1}}{R_{\text {esp_b }}+1}\right)}
$$

onde $K_{1}(774.89$ e 480.89$)$ e $K_{2}(1321.08$ e 1201.14) para as bandas 10 e 11 , respectivamente, são os coeficientes de conversão para o satélite L8. A média de $T_{b}$ para as duas bandas foi considerada a temperatura de brilho da Figura 2 .

Os resultados para o albedo planetário e temperatura de brilho foram corrigidas atmosfericamente para obtenção do albedo $\left(\alpha_{0}\right)$ e temperatura $\left(T_{0}\right)$ da superfície através de equações de regressão obtidas de medições simultâneas em campo e por sensoriamento remoto na região semiárida brasileira (TEIXEIRA et al. 2008; TEIXEIRA et al., 2009a; TEIXEIRA, 2010, TEIXEIRA et al., 2014).

O índice da Diferença da Vegetação Normalizado (NDVI) é um indicador relacionado com as condições hídricas e de vegetação obtido como:

$$
N D V I=\frac{\alpha p_{(i p)} \alpha p_{(v e r m)}}{\alpha p_{(i p)}+\alpha p_{(v e r m)}}
$$

onde $\alpha p_{(i p)}$ e $\alpha p_{(v e r m)}$ representam, respectivamente, os valores de albedo planetário no infravermelho próximo e no vermelho do espectro solar, os quais para o L8 foram calculados das bandas B5 e B4, respectivamente.

Os valores instantâneos da razão da evapotranspiração atual (ET) para a de referência $\left(E T_{0}\right)$ foram modelados e multiplicados pelas grades de $E T_{0}$ das estações agrometeorológicas para estimativa da ET em larga escala:

$$
\frac{E T}{E T_{0}}=\exp \left[c+d\left(\frac{T_{0}}{\alpha_{0} N D V I}\right)\right]
$$

onde c e d são os coeficientes de regressão, os quais foram 1.8 e -0.008 , respectivamente, para as condições semiáridas do Brasil (TEIXEIRA et al., 2013, TEIXEIRA et al., 2014).

A Equação 6não se aplica em corpos d'água (NDVI $<0)$. Então, o conceito da evapotranspiração de equilíbrio $\left(E T_{e q}\right)$ é adotado para estas condições no algoritmo SAFER (RAUPACH, 2001), aplicando-se funções condicionais para os valores negativos de NDVI:

$$
E T_{e q}=0,035\left(\frac{s\left(R_{n}-G\right)}{s+\gamma}\right)
$$

onde s é a inclinação da curva de relação entre a pressão de saturação do vapor d'água para a $T_{a}$, G é o fluxo de calor no interior da superfície e $\gamma$ é a constante psicrométrica. Nestas circunstâncias, não há restrição de umidade na superfície para os fluxos hídricos, sendo estes limitados apenas pela energia disponível.

Os valores diários de $R_{n}$ foram obtidos através da equação de Slob:

$$
R_{n}=\left(1-\alpha_{0}\right) R_{G}-a_{L} \tau_{s w}
$$

onde o coeficiente $a_{L}$ foi espacialmente distribuído através de sua relação com $T_{a}$ (TEIXEIRA et al. 2008).

Para os valores diários de $\mathrm{G}$, a seguinte equação foi aplicada:

$$
\frac{G}{R_{n}}=a_{G} \exp \left(b_{G} \alpha_{0}\right)
$$

onde $a_{G}$ e $b_{G}$ são coeficientes de regressão considerados como 3.98 e -25.47, respectivamente, para as condições semiáridas brasileiras (TEIXEIRA et al. 2013. TEIXEIRA et al. 2014).

Nos cálculos da BIO, o modelo da radiação de Monteith foi aplicado (TEIXEIRA et al., 2009b; TEIXEIRA et al. 2013):

$$
B I O=\varepsilon_{\max } E_{f} R F A_{a b s} 0,864
$$

onde $\epsilon \max$ é a máxima eficiência de uso da radiação, a qual depende se as espécies são C3 ou C4; A fração evaporativa $\left(E_{f}\right)$ é o fluxo de calor latente $(\gamma E)$ obtido pela transformação da ET em unidades de energia, pela energia disponível $\left(R_{n}-\mathrm{G}\right) ; R F A_{a} b s$ é a radiação fotossinteticamente ativa absorvida e 0.864 é um fator de conversão de unidades. 
DETERMINAÇÃO DA PRODUTIVIDADE DA ÁGUA COM IMAGENS LANDSAT 8 NA REGIÃO SEMIÁRIDA DO BRASIL

Tabela 1: Parâmetros radiométricos para obtenção do albedo planetário ( $\left.\alpha_{p}\right)$ para cada banda (b) das imagens do Landsat 8 (L8): comprimentos de onda - $\lambda_{b}$; radiação solar média no topo da atmosfera $-R a_{b}$; e os pesos $-w_{b}$.

\begin{tabular}{cccccccc}
\hline Parâmetro & B1 & B2 & B3 & B4 & B5 & B6 & B7 \\
\hline$\lambda_{b}(\mu m)$ & $0,43-0,45$ & $0,45-0,51$ & $0,53-0,59$ & $0,64-0,67$ & $0,85-0,88$ & $1,57-1,65$ & $2,11-2,29$ \\
$R a_{b}\left(W m^{-2} \mu m^{-1}\right)$ & 1718,8 & 1810,4 & 1741,7 & 1558,3 & 962,5 & 206,3 & 68,8 \\
$w_{b}$ & 0,10 & 0,31 & 0,30 & 0,13 & 0,08 & 0,05 & 0,04 \\
\hline
\end{tabular}

A $R F A_{a} b s$ foi obtida em função do NDVI e da radiação fotossinteticamente ativa incidente $\left(R F A_{i} n c\right)$, que por sua vez foi estimada como uma fração de $R_{G}$ $(44 \%)$ :

$$
R A F_{a b s}=(f * N D V I+g) R A F_{i n c}
$$

onde os coeficientes $\mathrm{f}$ e $\mathrm{g}$ foram considerados 1.257 e -0.161 , respectivamente (BASTIAANSSEN; ALI 2003).

A produtividade da água (PA) baseada na ET Teixeira et al. (2009b), Teixeira et al. (2013) foi então considerada como:

$$
P A=\frac{B I O}{E T}
$$

Para a classificação da vegetação em culturas irrigadas e vegetação natural, o modelo SUREAL foi aplicado:

$$
r_{s}=\exp \left[h\left(\frac{T_{0}}{\alpha_{0}}\right)(1-N D V I)+i\right]
$$

onde h e i são coeficientes de regressão, respectivamente 0.04 e 2.72 para as condições semiáridas do Brasil (Teixeira, 2010; Teixeira et al., 2013). Valores de $r_{s}$ abaixo de $800 \mathrm{~s} \mathrm{~m}^{-1}$ e NDVI acima ou igual a 0.4 foram considerados culturas irrigadas, enquanto que valores de $r_{s}$ entre 1000 e $10000 \mathrm{~s} \mathrm{~m}^{-1}$ e NDVI abaixo de 0.4 foram considerados vegetação natural. $\mathrm{O}$ valor acima desta faixa foi incluído para considerar construções humanas.

\section{RESULTADOS E DISCUSSÃO}

\subsection{Parâmetros climáticos}

Os parâmetros de produtividade da água são afetados pelas condições climáticas, podendo estas serem analisadas através das tendências da $R_{G}$, da precipitação (P) e da evapotranspiração de referência $\left(E T_{0}\right)$, nos períodos que influenciam os resultados das imagens. Estas tendências são apresentadas na Figura 3 , desde o começo da estação chuvosa em 2013 ao final da estação seca em 2014 em termos de dia juliano (DJ), abrangendo as condições termo hídricas prévias, durante e depois das datas de aquisição das imagens L8.

Pela Figura 2, percebe-se que P foi o parâmetro climático mais variável. Apesar da imagem DJ 025 do ano 2014 está dentro do período chuvoso, a chuva acumulada a partir do final de 2013 (DJ 305) até a data da imagem em 2014 (DJ 025) foi de apenas $85 \mathrm{~mm}$, enquanto que o total acumulado daí até DJ 153, imagem representante do período logo após as chuvas, foi de $280 \mathrm{~mm}$. Deste dia até o dia da imagem representante do período mais seco (DJ 265) a precipitação acumulada foi de $64 \mathrm{~mm}$. Pelos valores diários de $E T_{0}$, as maiores demandas atmosféricas ocorreram entre os DJ 305-346 de 2013 e 021-045 de 2014, quando chegaram a valores superiores a 7,0 $\mathrm{mm} \mathrm{dia}^{-1}$. Nestas situações o sol esteve em torno de sua posição zenital com céu apresentando baixa cobertura de nuvens. Os valores médios diários de $R_{G}$, acima de $25,0 \mathrm{MJ} \mathrm{m}^{-2} \mathrm{dia}^{-1}$, ocorreram do final de 2013 para o início de 2014 (DJ 345 e 010 , respectivamente), e abaixo de $15 \mathrm{MJ} \mathrm{m}^{-2}$ $\mathrm{dia}^{-1}$, mais frequentemente no meio do ano de 2014, com os diferentes níveis relacionados com a posição astronômica do sol e com a cobertura de nuvens. Nas condições de elevados valores de P, $E T_{0}$ e $R_{G}$, todos agros-ecossistemas, vegetação natural e culturas irrigadas foram favorecidos para elevados valores da ET, BIO e PA.

\subsection{Parâmetros obtidos por sensoriamento remoto}

As variáveis hídricas e de vegetação dos diferentes agros-ecossistemas dependem do albedo da superfície $\left(\alpha_{0}\right)$, do Índice da Diferença da Vegetação Normalizada (NDVI) e da temperatura da superfície $\left(T_{0}\right)$. O primeiro parâmetro, o $\alpha_{0}$, determina a radiação de ondas curtas que retorna da superfície para a baixa atmosfera; o NDVI é um parâmetro chave que indica as condições de cobertura e umidade do solo; e a radiação de ondas longas emitida pela superfície é diretamente proporcional a $T_{0}$. Estes então são os parâmetros básicos obtidos por sensoriamento remoto no momento da passagem do satélite Landsat 8 para a modelagem da PA em larga escala.

A distribuição espacial dos parâmetros de entrada 


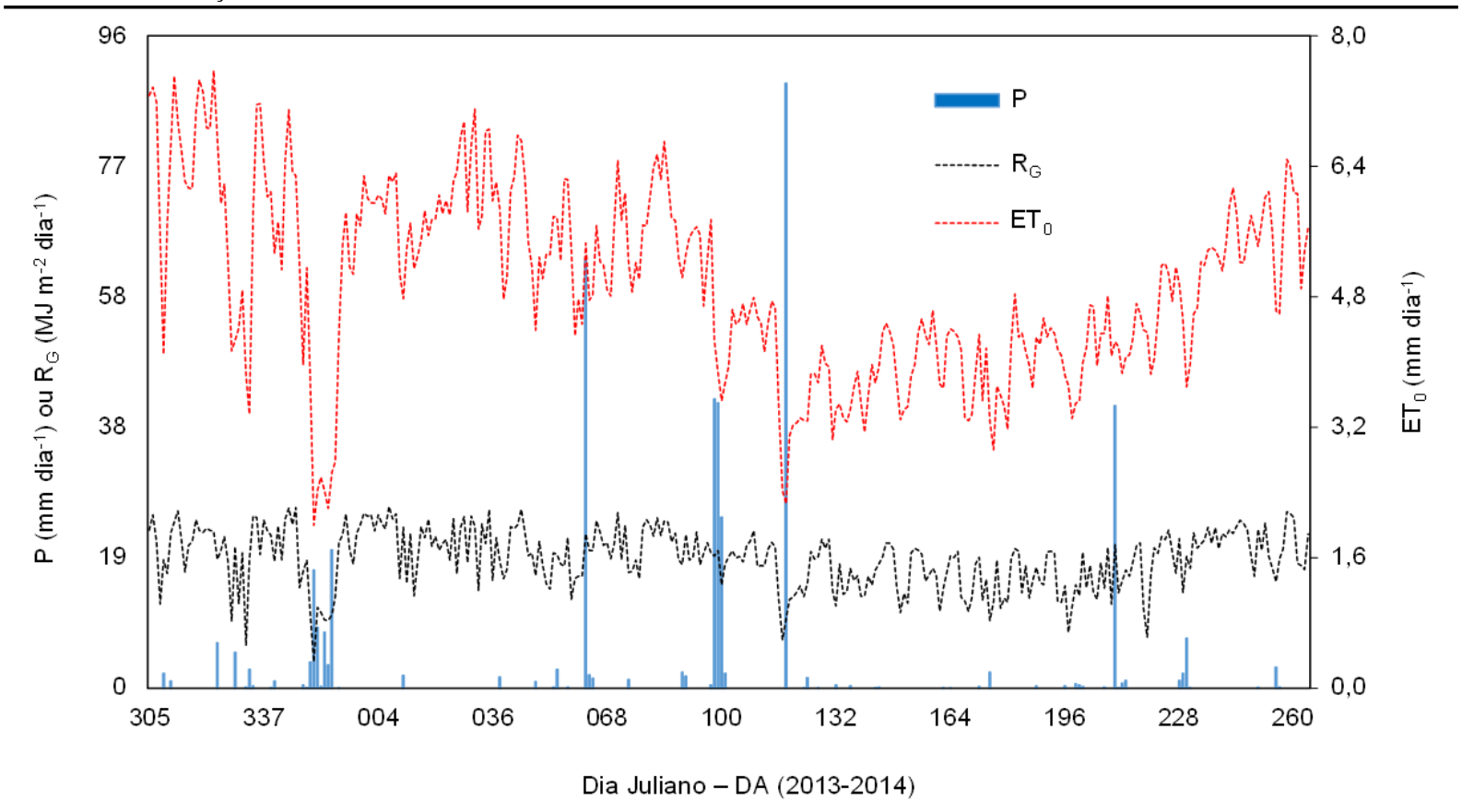

Figura 3: Valores diários da precipitação $(\mathrm{P})$, radiação solar global incidente $\left(R_{G}\right)$ e evapotranspiração de referência $\left(E T_{0}\right)$, envolvendo os dias julianos (DJ) do começo da estação chuvosa em novembro/2013 ao final da estação seca em setembro/2014, obtidos da estação agrometeorológicas Santa Helena (ver Figura 1, no município de Juazeiro/BA, região Nordeste do Brasil.

da modelagem dos componentes da PA, obtidos através das medições do satélite L8, envolvendo diferentes condições termo hídricas no ano de 2014 na região semiárida do município de Juazeiro/BA, Nordeste do Brasil, são apresentadas na Figura 4.

Nos agros-ecossistemas mistos da região semiárida brasileira, as superfícies escuras da Caatinga apresentam baixos valores de $\alpha_{0}$, absorvendo mais radiação solar que aquelas mais claras, dependendo ainda das condições de umidade do solo (van Dijk et al., 2004; Li et al., 2006; Teixeira et al., 2008). Os valores mais elevados de $\alpha_{0}$ ocorreram na imagem de DJ 265, representativa do período mais seco do ano. Isto se deve ao efeito conjunto de baixa umidade do solo, nuvens escarças e posição astronômica do sol, favorecendo elevados níveis de $R_{G}$. Entretanto, as variações espaciais de $\alpha_{0}$ foram homogêneas durante os diferentes períodos do ano com desvios padrões (DV) de 0.02 em todas as imagens, sem grandes distinções entre as culturas irrigadas e vegetação natural. Monteith e Unsworth (1990) reportaram $\alpha_{0}$ entre 0.15 e 0.26 para vegetações naturais tropicais, entretanto os valores dos ecossistemas mistos do estudo corrente são mais elevados que aqueles encontrados para regiões tropicais mais húmidas (OGUNTOYINBO, 1970, PINKER; THOMPSON; ECK, 1980, SHUTTLEWORTH, 1988). A re- lação de $\alpha_{0}$ com as condições ambientais e de umidade está de acordo com outros estudos mais recentes (LOBELL; ASNER, 2002, DIJK; BRUIJNZEEL; SCHELLEKENS, 2004; LI et al., 2006; TEIXEIRA et al., 2014). Através de medições de campo na Caatinga, Teixeira et al. (2008) reportaram a dependência de $\alpha_{0}$ com as condições das chuvas, variando entre 0.10 e 0.18 , similares a várias áreas representativas deste natural ecossistema na Figura 4

As distinções dos valores de NDVI entre as culturas irrigadas e a vegetação natural são bem mais visíveis do que no caso de $\alpha_{0}$, com as maiores diferenças entre os agros-ecossistemas ocorrendo nas condições mais secas no final de setembro (DJ 265). A média dos pixels mais elevada ocorreu em junho (imagem de DJ 153), devido ao fato de que, por um lado, as águas das chuvas consumidas pela Caatinga promoveram forte desenvolvimento vegetativo (Figuras $2 \mathrm{e} 4$, , e por outro, as culturas irrigadas estão bem desenvolvidas devido a um suprimento adicional regular de água via irrigação. Os valores mais baixos de NDVI ocorreram em setembro (DJ 265), quando a umidade na zona das raízes das espécies naturais é baixa, promovendo condições de senescência. Percebe-se que nas áreas de Caatinga os valores de NDVI ficam abaixo de 0.40 , similarmente aos reportados por Teixeira et al. (2014) com o uso de imagens 

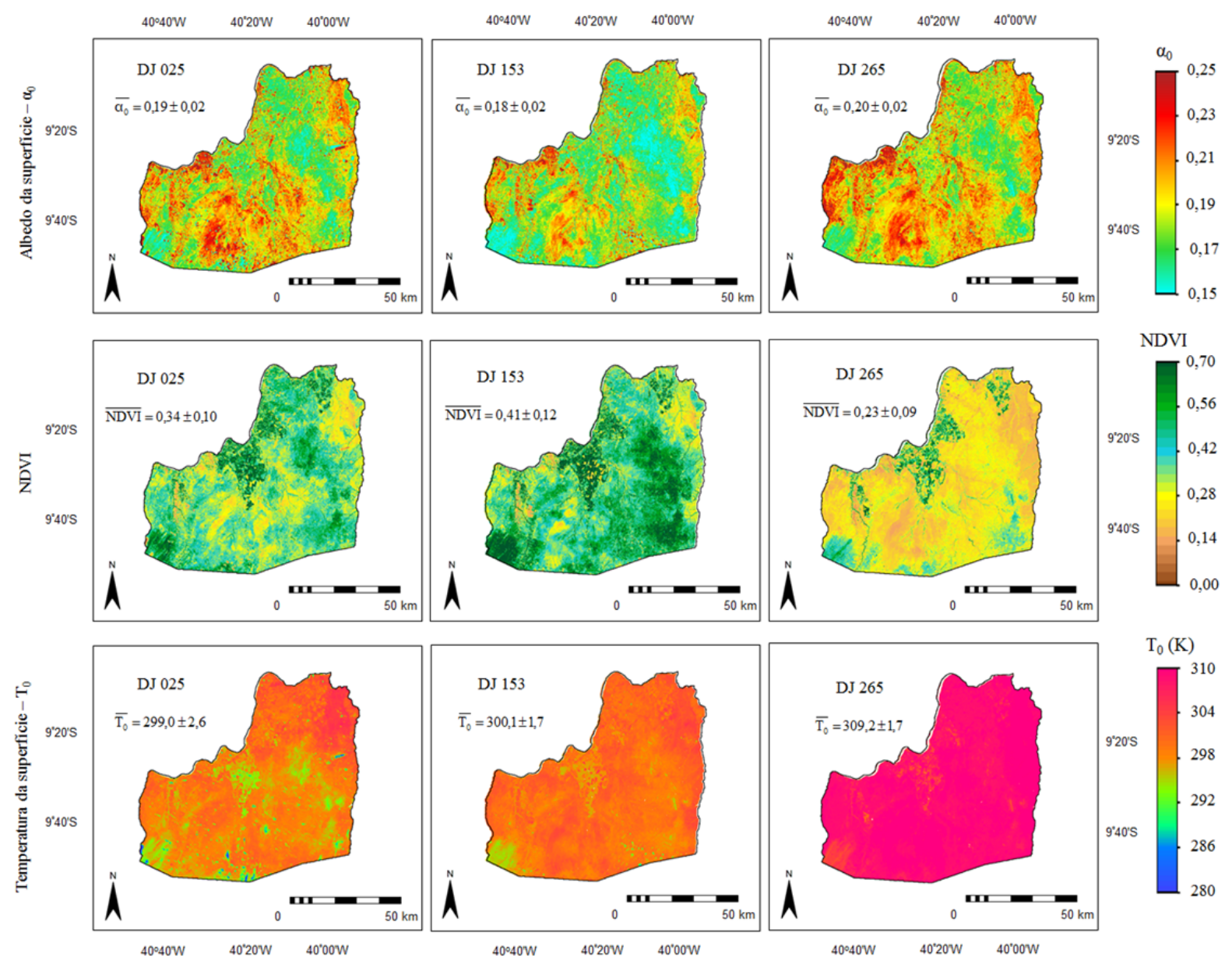

Figura 4: Distribuição espacial dos parâmetros de entrada para a modelagem da produtividade da água, obtidos das medições do satélite L8, sob as diferentes condições termo hídricas do ano de 2014, na região semiárida do município de Juazeiro/BA, Nordeste do Brasil. Albedo da superfície $\left(\alpha_{0}\right)$; Índice da Diferença da Vegetação Normalizado (NDVI); e temperatura da superfície $\left(T_{0}\right)$. DJ é o dia Juliano e as barras significam valores médios dos pixels. 
DETERMINAÇÃO DA PRODUTIVIDADE DA ÁGUA COM IMAGENS LANDSAT 8 NA REGIÃO SEMIÁRIDA DO BRASIL

Landsat 5 e MODIS na região semiárida do Brasil.

$T_{0}$ afeta a energia disponível, pela interferência no balanço de radiação de ondas longas. Valores mais baixos ocorreram nas culturas irrigadas comparando com as áreas mais secas de vegetação natural. Entretanto, apesar de $T_{0}$ diferenciar os agros-ecossistemas melhor com relação à de $\alpha_{0}$, esta distinção não é tão forte como no caso do NDVI. Uma das razões para este fato é a menor resolução espacial da banda termal $(100 \mathrm{~m})$ em comparação com a faixa do visível e infravermelho próximo $(30 \mathrm{~m})$ do sensor L8. O período de maiores valores de $T_{0}$ coincidiu com aqueles de níveis mais elevados de $R_{G}$, representado pela imagem DJ 265, enquanto que os menores aconteceram na estação chuvosa (DJ 025).

Classificando-se os agros-ecossistemas através do modelo SUREAL, os valores médios dos pixels para $\alpha_{0}$, NDVI e $T_{0}$ em culturas irrigadas (CI) e vegetação natural (VN), são mostrados na Tabela 2 .

O parâmetro $\alpha_{0}$ representa a razão da radiação que é refletida $\left(R_{R}\right)$ para a incidente $\left(R_{G}\right)$. Embora os valores de $R_{R}$ sejam influenciados pelos de $R_{G}$, os primeiros são também afetados pelas condições de umidade e cobertura do solo. Pela Tabela 2 e Figura 4 percebem-se os maiores valores de $\alpha_{0}$ no final de setembro (DJ 265), entretanto as maiores diferenças entre culturas irrigadas (CI) e a vegetação natural (VN), de até 0.02 , aconteceram em janeiro (DJ 025). No período representado pela imagem de DJ 265 ocorreram também as maiores diferenças nos valores de NDVI, sendo em média aqueles para a classe CI o dobro daqueles para a classe VN. Os maiores valores de $T_{0}$ também são para as condições de níveis mais elevados de $R_{G}$ no final de setembro, porém com aqueles para a classe CI sendo $99 \%$ daqueles para a classe VN. Esta diferença evidencia que a irrigação, apesar de poder causar competição por água com outros setores não agrícolas, ameniza o efeito de elevadas temperaturas em larga escala nas condições semiáridas brasileiras, o que deve ser considerado na modelagem de cenários futuros de mudanças climáticas.

As variações espaciais e temporais de $\alpha_{0}$, NDVI e $T_{0}$ na Caatinga, podem ser atribuídas à heterogeneidade nos níveis de $R_{G}$ e das condições de umidade e cobertura do solo, como consequência do movimento aparente do sol, nebulosidade e regime de precipitações. Entretanto, em condições de irrigação, além destas influências, estes parâmetros, obtidos por sensoriamento remoto, são também afetados pelos manejos de água e das culturas, influenciando a partição da energia entre os diferentes agros-ecossistemas.

\subsection{Produtividade da água em larga escala}

A Figura 5 apresenta a distribuição espacial dos valores diários da ET, BIO e PA, sob as diferentes condições termo hídricas do ano de 2014, no município de Juazeiro-BA, Nordeste do Brasil.

As variações espaciais e temporais dos parâmetros de produtividade da água são evidentes na Figura 5 . principalmente quando se compara os períodos de condições de solo mais úmido com aqueles mais secos, representados respectivamente pelas imagens de DJ $153 \mathrm{e}$ 265 , onde os pixels de maiores valores de ET, BIO e PA representam as culturas agrícolas bem irrigadas. Os valores mais elevados de todos os parâmetros ocorreram após o período chuvoso (DJ 153), quando as precipitações acumuladas favoreceram as espécies da Caatinga, enquanto que além do fornecimento natural de água pelas chuvas, as culturas agrícolas estavam bem irrigadas. A menor média dos pixels ocorreu no período mais seco (DJ 265), devido ao baixo desenvolvimento das espécies de vegetação natural, que ocupa a maior área do município.

Por um lado, as maiores variações da ET e da $\mathrm{BIO}$, observados pelos maiores valores de desvio padrão (DP), ocorreram nas condições mais úmidas (DJ 025 e 153). Por outro lado, como a Caatinga cobre uma maior área, o período mais seco (DJ 265) apresenta os menores valores de DP, devido ao fato das espécies naturais estarem em estado de dormência. Os estômatos se fecham durante estas últimas condições, limitando a transpiração e a fotossíntese, e em geral, as irrigações uniformes das culturas são diárias, favorecendo elevadas taxas da ET nas parcelas bem irrigadas.

Os valores máximos de PA para as culturas irrigadas quando multiplicados pelo índice de colheita (IH) fornecem a produtividade da água das culturas (PAC). Valores de IH foram em torno de 0.60 e 0.80 para parreirais e mangueiras nas condições semiáridas do Brasil obtidos com a utilização de imagens Landsat 5 e 7 , os quais retornaram PAC em torno de 2.8 e $3.4 \mathrm{~kg}$ $\mathrm{m}^{-3}$, respectivamente (TEIXEIRA et al. 2009b). Estes estudos prévios corroboram com o estudo corrente, considerando-se os maiores valores dos componentes da produtividade da água na Figura 5, correspondentes às áreas de cobertura de vários videiras e pomares de mangueiras.

A Tabela 3 apresenta os valores médios e desvios padrões (DP) dos parâmetros de produtividade da água (PA) para culturas irrigadas (CI) e vegetação natural (VN), após classificação com o modelo SUREAL.

Extraindo-se apenas vegetação de todo o município de Juazeiro, e considerando ambos os ecossistemas, culturas irrigadas e vegetação natural, os maiores valo- 
Tabela 2: Valores médios e desvios padrões dos parâmetros de entrada para a modelagem da produtividade da água (PA) em larga escala, nas condições semiáridas do município de Juazeiro/BA, Nordeste do Brasil, no ano de 2014. Albedo da superfície $\left(\alpha_{0}\right)$; Índice da Diferença da Vegetação Normalizado (NDVI); e temperatura da superfície $\left(T_{0}\right)$.

\begin{tabular}{ccccccc}
\hline DJ/Média & \multicolumn{2}{c}{$\alpha \_0(-)$} & \multicolumn{2}{c}{ NDVI (-) } & \multicolumn{2}{c}{$T \_0(\mathbf{K})$} \\
\hline DJ & CI & VN & CI & VN & CI & VN \\
\hline 025 & $0,18 \pm 0,01$ & $0,20 \pm, 0,02$ & $0,54 \pm, 0,09$ & $0,30 \pm, 0,06$ & $297,6 \pm, 2,3$ & $298,8 \pm, 1,7$ \\
153 & $0,18 \pm 0,01$ & $0,19 \pm 0,02$ & $0,57 \pm, 0,11$ & $0,31 \pm, 0,06$ & $298,7 \pm, 1,6$ & $301,1 \pm, 1,4$ \\
265 & $0,19 \pm 0,01$ & $0,20 \pm 0,02$ & $0,55 \pm, 0,09$ & $0,21 \pm, 0,05$ & $305,8 \pm, 1,9$ & $309,4 \pm, 1,5$ \\
Média & $0,18 \pm 0,01$ & $0,20 \pm 0,02$ & $0,55 \pm, 0,10$ & $0,27 \pm, 0,06$ & $300,7 \pm, 1,9$ & $303,1 \pm, 1,5$ \\
\hline \multicolumn{7}{c}{$*$ CI - Culturas irrigadas; VN - Vegetação Natural; DJ - Dia Juliano. }
\end{tabular}
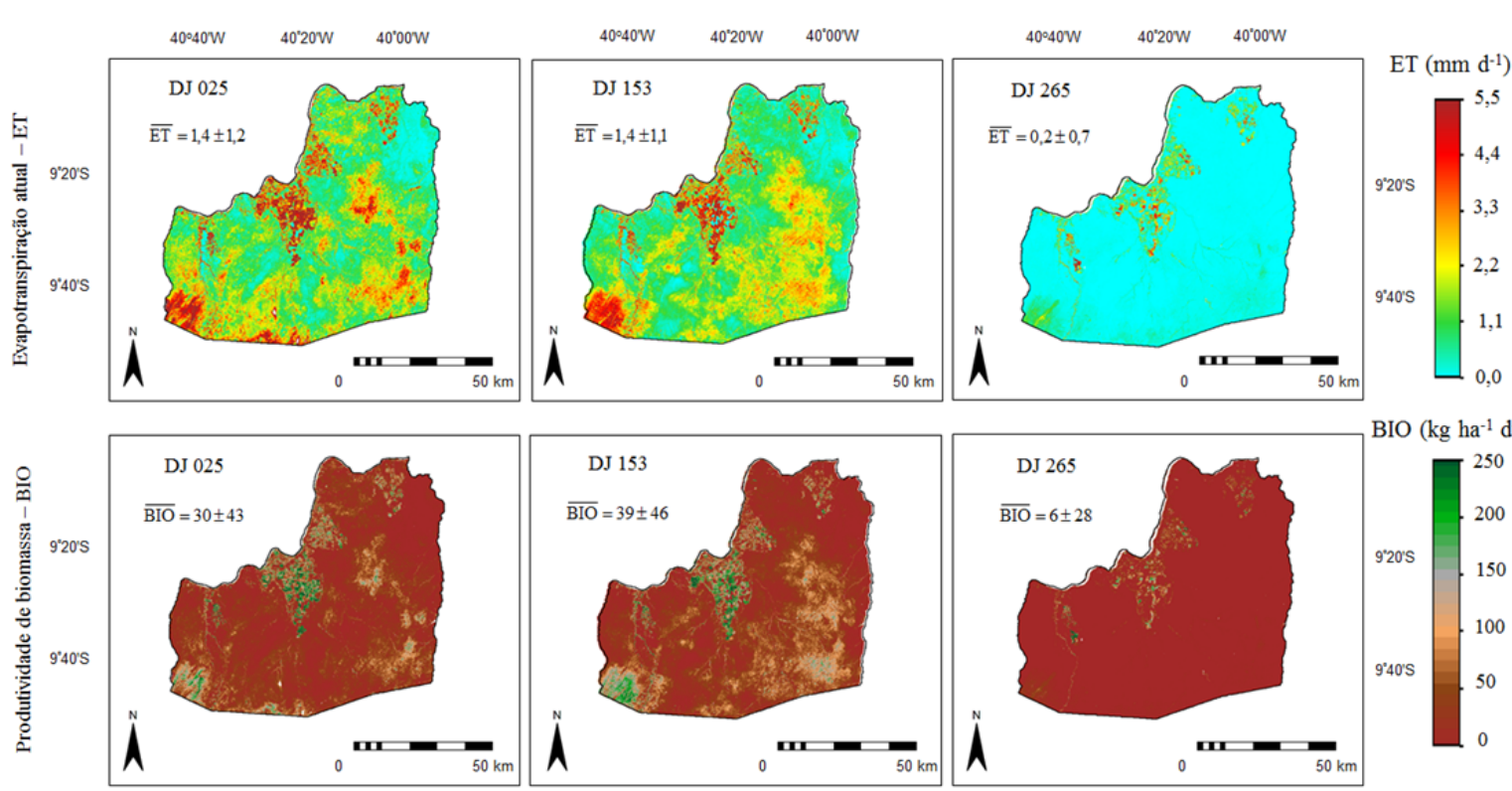

$\mathrm{BIO}\left(\mathrm{kg} \mathrm{ha}^{-1} \mathrm{~d}^{-1}\right)$
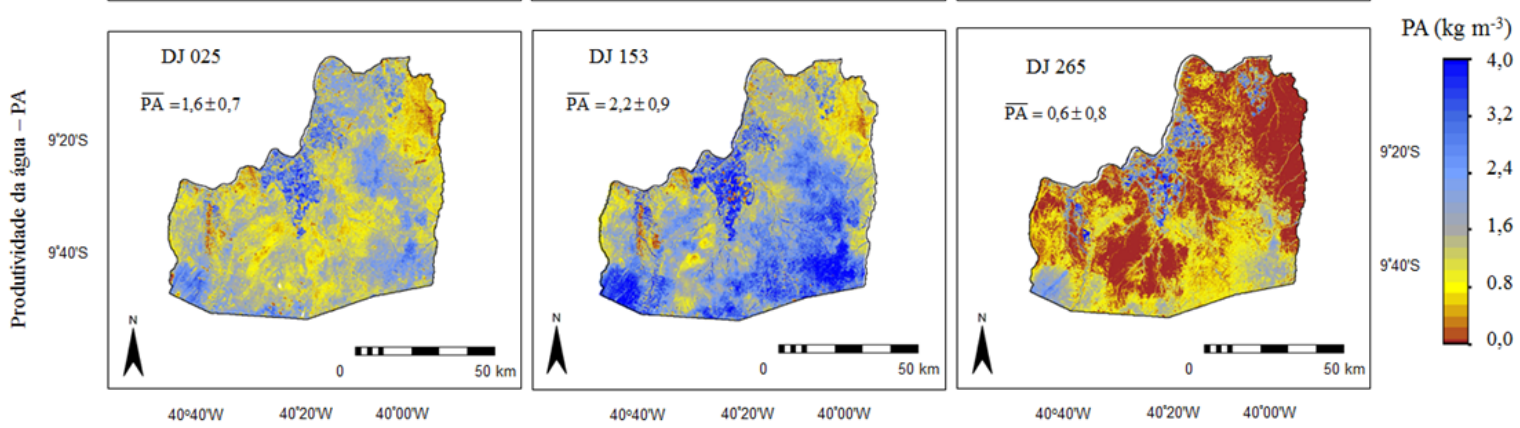

Figura 5: Distribuição espacial dos valores diários dos parâmetros da produtividade da água em larga escala, sob as diferentes condições termo hídricas do ano de 2014, na região semiárida do município de Juazeiro/BA, Nordeste do Brasil. Evapotranspiração atual (ET); produção de biomassa (BIO); e produtividade da água (PA) baseada na ET. DJ é o dia Juliano e as barras significam valores médios dos pixels. 
DETERMINAÇÃO DA PRODUTIVIDADE DA ÁGUA COM IMAGENS LANDSAT 8 NA REGIÃO SEMIÁRIDA DO BRASIL

Tabela 3: Valores médios diários e desvios padrões dos parâmetros de produtividade da água em culturas e irrigadas e vegetação natural, sob diferentes condições termo hídricas na região semiárida do município de Juazeiro-BA, Nordeste do Brasil. Evapotranspiração atual (ET); produção de biomassa (BIO); e produtividade da água baseada da ET (PA).

\begin{tabular}{ccccccc}
\hline DJ/Média & \multicolumn{2}{c}{ ET $\left(\mathrm{mm} \mathrm{d}^{-1}\right)$} & \multicolumn{2}{c}{ BIO $\left(\mathrm{kg} \mathrm{ha}^{-1} d^{-1}\right)$} & \multicolumn{2}{c}{ PA $\left(\mathrm{kg} \mathrm{m}^{-3}\right)$} \\
\hline DJ & CI & VN & CI & VN & CI & VN \\
\hline 025 & $3,8 \pm 1,6$ & $0,9, \pm 0,6$ & $124 \pm, 75$ & $13 \pm, 11$ & $3,0 \pm, 0,7$ & $1,3 \pm, 0,4$ \\
153 & $2,9 \pm 1,2$ & $0,6 \pm 0,4$ & $107 \pm, 64$ & $10 \pm, 8$ & $3,4 \pm, 0,8$ & $1,4 \pm, 0,5$ \\
265 & $3,1 \pm 1,4$ & $0,1 \pm 0,1$ & $115 \pm, 77$ & $1 \pm, 2$ & $3,4 \pm, 0,7$ & $0,5 \pm, 0,6$ \\
Média & $3,3 \pm 1,4$ & $0,5 \pm 0,4$ & $115 \pm, 72$ & $8 \pm, 7$ & $3,3 \pm, 0,7$ & $1,1 \pm, 0.5$ \\
\hline \multicolumn{4}{r}{${ }^{*}$ CI - Culturas irrigadas; VN - Vegetaçăo Natural; DJ - Dia Juliano. }
\end{tabular}

res médios da ET e da BIO ocorreram no período chuvoso representado pelas imagens de DJ 025, enquanto que no caso da PA, os resultados da imagem DJ 153 apresentaram as maiores taxas, representando as condições logo após o período chuvoso. Considerando-se os valores de DP, o período de maior variação espacial da ET e da BIO foi durante a estação chuvosa (DJ 025) indicando diferentes condições de umidade do solo em vegetação natural e heterogeneidade nas fases fenológicas nas culturas irrigadas. Maior uniformidade para os parâmetros de produtividade da água ocorreu na vegetação natural, evidenciada pelos menores valores de DP quando comparados com aqueles nas condições de irrigação. Os valores da ET obtidos no polo agrícola Petrolina/Juazeiro foram similares aos encontrados por Freitas et al. (2016) no perímetro de Irrigação Jaíba, situado no Médio São Francisco, neste caso com valores entre chegando a $3.5 \pm 1.0 \mathrm{~mm} \mathrm{~d}^{-1}$. Os valores ligeiramente inferiores aos da Tabela 3 podem ser atribuídos à menor resolução de $250 \mathrm{~m}$ das imagens MODIS usadas para Jaíba.

\section{CONCLUSÕES}

O uso conjunto de imagens do satélite Landsat 8 e estações agrometeorológicas permitiram a quantificação em larga escala dos parâmetros da produtividade da água em diferentes condições termo hídricas do ano de 2014, considerando-se a mistura de agros-ecossistemas no município de Juazeiro-BA. As análises podem subsidiar um melhor entendimento da dinâmica destes parâmetros, considerando-se as mudanças climáticas e de uso da terra no semiárido brasileiro. Extraindo-se apenas as superfícies vegetadas do município, pôde-se concluir que o período chuvoso se destaca como o de maior evapotranspiração e produção de biomassa tanto para culturas irrigadas como para vegetação natural, porém os maiores valores da produtividade da água baseada na evapotranspiração ocorrem logo após as chuvas em ambos ecossistemas.

Foi demonstrado que a produtividade da água pode ser analisada a partir de medições instantâneas das radiações de ondas curtas e longas do Landsat 8, através da modelagem da razão da evapotranspiração atual para a de referência no momento da passagem do satélite, tendo-se disponível dados climáticos diários. A combinação de parâmetros obtidos por sensoriamento remoto com dados agrometeorológicos, destaca-se como muito útil para o monitoramento das condições da vegetação no semiárido brasileiro, contribuindo para a sustentabilidade da mistura de agros-ecossistemas, característica da região.

\section{AGRADECIMENTOS}

Ao Conselho Nacional de Pesquisa (CNPq), pelo aporte financeiro referente a projetos sobre produtividade da água.

\section{REFERÊNCIAS}

ALLEN, R. G.; PEREIRA, L. S.; RAES, D.; SMITH, M. et al. Crop evapotranspiration-guidelines for computing crop water requirements-fao irrigation and drainage paper 56. FAO, Rome, v. 300, n. 9, p. D05109, 1998.

BASTIAANSSEN, W. G.; ALI, S. A new crop yield forecasting model based on satellite measurements applied across the indus basin, pakistan. Agriculture, ecosystems \& environment, Elsevier, v. 94, n. 3, p. 321-340, 2003.

BASTIAANSSEN, W. G.; MENENTI, M.; FEDDES, R.; HOLTSLAG, A. A remote sensing surface energy balance algorithm for land (sebal). 1. formulation. Journal of hydrology, Elsevier, v. 212, p. 198-212, 1998. 
DETERMINAÇÃO DA PRODUTIVIDADE DA ÁGUA COM IMAGENS LANDSAT 8 NA REGIÃO SEMIÁRIDA DO BRASIL

CESCHIA, E.; BÉZIAT, P.; DEJOUX, J.-F.; AUBINET, M.; BERNHOFER, C.; BODSON, B.; BUCHMANN, N.; CARRARA, A.; CELLIER, P.; TOMMASI, P. D. et al. Management effects on net ecosystem carbon and ghg budgets at european crop sites. Agriculture, ecosystems \& environment, Elsevier, v. 139, n. 3, p. 363-383, 2010.

CLEUGH, H. A.; LEUNING, R.; MU, Q.; RUNNING, S. W. Regional evaporation estimates from flux tower and modis satellite data. Remote Sensing of Environment, Elsevier, v. 106, n. 3, p. 285-304, 2007.

DIJK, A. I. V.; BRUIJNZEEL, L. S.; SCHELLEKENS, J. Micrometeorology and water use of mixed crops in upland west java, indonesia. Agricultural and forest meteorology, Elsevier, v. 124, n. 1, p. 31-49, 2004.

KAMBLE, B.; KILIC, A.; HUBBARD, K. Estimating crop coefficients using remote sensing-based vegetation index. Remote Sensing, Multidisciplinary Digital Publishing Institute, v. 5, n. 4, p. 1588-1602, 2013.

LEIVAS, J. F.; TEIXEIRA, A. H. C.; BAYMA-SILVA, G.; RONQUIM, C. C.; REIS, J. B. R. da S. Biophysical indicators based on satellite images in an irrigated area at the são francisco river basin, brazil. In: INTERNATIONAL SOCIETY FOR OPTICS AND PHOTONICS. SPIE Remote Sensing. 2016. p. 99981N-99981N.

LI, S.-G.; EUGSTER, W.; ASANUMA, J.; KOTANI, A.; DAVAA, G.; OYUNBAATAR, D.; SUGITA, M. Energy partitioning and its biophysical controls above a grazing steppe in central mongolia. Agricultural and Forest Meteorology, Elsevier, v. 137, n. 1, p. 89-106, 2006.

LOBELL, D. B.; ASNER, G. P. Moisture effects on soil reflectance. Soil Science Society of America Journal, Soil Science Society, v. 66, n. 3, p. 722-727, 2002 .

MIRALLES, D.; HOLMES, T.; JEU, R. D.; GASH, J.; MEESTERS, A.; DOLMAN, A. Global land-surface evaporation estimated from satellite-based observations. Hydrology and Earth System Sciences, Copernicus GmbH, v. 15, n. 2, p. 453, 2011.

MOLDEN, D.; FRENKEN, K.; BARKER, R.; FRAITURE, C. d.; MATI, B.; SVENDSEN, M.; SADOFF, C.; FINLAYSON, C. M.; ATTAPATU, S.; GIORDANO, M. et al. Trends in water and agricultural development. International Water Management Institute, 2007.
MONTEITH, J. Solar radiation and productivity in tropical ecosystems. Journal of applied ecology, JSTOR, v. 9, n. 3, p. 747-766, 1972.

MONTEITH, J.; UNSWORTH, M. Principles of environmental physics.,(Arnold: London). : UK, 1990.

NAGLER, P. L.; GLENN, E. P.; NGUYEN, U.; SCOTT, R. L.; DOODY, T. Estimating riparian and agricultural actual evapotranspiration by reference evapotranspiration and modis enhanced vegetation index. Remote Sensing, Multidisciplinary Digital Publishing Institute, v. 5, n. 8, p. 3849-3871, 2013.

OGUNTOYINBO, J. Reflection coefficient of natural vegetation, crops and urban surfaces in nigeria. Quarterly Journal of the Royal Meteorological Society, Wiley Online Library, v. 96, n. 409, p. 430-441, 1970.

PINKER, R. T.; THOMPSON, O. E.; ECK, T. The albedo of a tropical evergreen forest. Quarterly Journal of the Royal Meteorological Society, Wiley Online Library, v. 106, n. 449, p. 551-558, 1980.

PÔÇAS, I.; CUNHA, M.; PEREIRA, L. S.; ALLEN, R. G. Using remote sensing energy balance and evapotranspiration to characterize montane landscape vegetation with focus on grass and pasture lands. International Journal of Applied Earth Observation and Geoinformation, Elsevier, v. 21, p. 159-172, 2013.

RAUPACH, M. Combination theory and equilibrium evaporation. Quarterly Journal of the Royal Meteorological Society, Wiley Online Library, v. 127, n. 574, p. 1149-1181, 2001.

SHUTTLEWORTH, W. J. Evaporation from amazonian rainforest. Proceedings of the Royal Society of London B: Biological Sciences, The Royal Society, v. 233, n. 1272, p. 321-346, 1988.

TANG, Q.; ROSENBERG, E.; LETTENMAIER, D. Use of satellite data to assess the impacts of irrigation withdrawals on upper klamath lake, oregon. Hydrology and Earth System Sciences, Copernicus GmbH, v. 13, n. 5, p. 617-627, 2009.

TEIXEIRA, A.; BASTIAANSSEN, W.; AHMAD, M.-u.-D.; MOURA, M. d.; BOS, M. Analysis of energy fluxes and vegetation-atmosphere parameters in irrigated and natural ecosystems of semi-arid brazil. Journal of Hydrology, Elsevier, v. 362, n. 1, p. 110-127, 2008.

TEIXEIRA, A. d. C.; BASTIAANSSEN, W.; AHMAD, M.-u.-D.; BOS, M. Reviewing sebal input 
parameters for assessing evapotranspiration and water productivity for the low-middle sao francisco river basin, brazil: Part a: Calibration and validation. agricultural and forest meteorology, Elsevier, v. 149, n. 3, p. 462-476, 2009.

Reviewing sebal input parameters for assessing evapotranspiration and water productivity for the low-middle sao francisco river basin, brazil: Part b: Application to the large scale. agricultural and forest meteorology, Elsevier, v. 149, n. 3, p. 477-490, 2009.

TEIXEIRA, A. H. d. C. Determining regional actual evapotranspiration of irrigated crops and natural vegetation in the são francisco river basin (brazil) using remote sensing and penman-monteith equation. Remote Sensing, Molecular Diversity Preservation International, v. 2, n. 5, p. 1287-1319, 2010.

TEIXEIRA, A. H. d. C.; HERNANDEZ, F. B. T.; LOPES, H.; SCHERER-WARREN, M.; BASSOI, L. H. A comparative study of techniques for modeling the spatiotemporal distribution of heat and moisture fluxes at different agroecosystems in brazil. Remote Sensing of Energy Fluxes and Soil Moisture Content, CRC Press, p. 169-191, 2014.

TEIXEIRA, A. H. d. C.; SCHERER-WARREN, M.; HERNANDEZ, F. B.; ANDRADE, R. G.; LEIVAS, J. F. Large-scale water productivity assessments with modis images in a changing semi-arid environment: a brazilian case study. Remote Sensing, Multidisciplinary Digital Publishing Institute, v. 5, n. 11, p. 5783-5804, 2013.

TEIXEIRA, A. H. D. C. T. Water productivity assessments from field to large scale: a case study in the Brazilian semi-arid region. : Lambert Academic Publishing, 2009.

VANHELLEMONT, Q; RUDDICK, K. Turbid wakes associated with offshore wind turbines observed with landsat 8. Remote Sensing of Environment, Elsevier, v. 145, p. 105-115, 2014. 